\title{
Advanced Studies for Resolving Nonlinear Dynamic Systems Involving Complexity Based on Systems Thinking for Non-Physicists
}

\author{
Deok-Soo Cha', Kim Kyoung-II² \\ ${ }^{1}$ Eho Technology Co. Seoul, S. Korea, Soong-Sil University, Seoul, Korea \\ ${ }^{2}$ Electrical Power Division, Seoul in S. Korea, Chung-Ang University, Seoul, Korea \\ Email: chdsoo@hotmail.com,kb01155@naver.com
}

How to cite this paper: Cha, D.-S. and Kyoung-Il, K. (2021) Advanced Studies for Resolving Nonlinear Dynamic Systems Involving Complexity Based on Systems Thinking for Non-Physicists. Open Journal of Applied Sciences, 11, 985-996.

https://doi.org/10.4236/ojapps.2021.118071

Received: July 22, 2021

Accepted: August 28, 2021

Published: August 31, 2021

Copyright (C) 2021 by author(s) and Scientific Research Publishing Inc. This work is licensed under the Creative Commons Attribution International License (CC BY 4.0).

http://creativecommons.org/licenses/by/4.0/

\section{(c) (i) Open Access}

\begin{abstract}
There are many examples of nonlinear dynamics, such as food chains or thermodynamic systems within closed-loop systems. In modern physics, these problems have been resolved based on logical thinking by using the chaos theory in statistical physics, which was arranged by classical physicists in the $17^{\text {th }}$ century. However, this is a significantly erroneous problem because, in engineering science, nonlinear dynamics must be resolved and cleared using systems analysis theory based on systems thinking. It is the main concept in a new solution that is studied through interdisciplinary research; it is needed to introduce control theory into physics. In 2015, on the behalf of physicists, the author successfully resolved and achieved a new solution, which is a significant achievement in modern science. Unfortunately, physicists have not welcomed it because it is disadvantageous to them, similar to the Copernican theory. So, they themselves became outsiders. If so, non-physicists need to follow their chaos theory in physics unless they do not clarify their solution; moreover, non-physicists on behalf of physicists can use their own new solution without risk. Hence, all scientists need to learn the systems analytic method in engineering.
\end{abstract}

\section{Keywords}

Nonlinear Dynamics, Control Theory, MATLAB, Systems Thinking

\section{Introduction}

This article details an advanced study on a new solution for resolving nonlinear dynamic systematic problems involving complexity [1] within closed-loop systems, such as the food chains in ecosystems, daily prices in the stock market, or 
daily weather in thermo-dynamics. Meanwhile, modern science has resolved these problems using chaos theory in statistical physics based on determinism; however, it is not fully resolved yet. While the chaos theory was arranged by classical physicists in the $17^{\text {th }}$ century (not modern physicists), and they approached it based on logical thinking and resolved them using logical solutions such as the previous chaos theory. However, it is imperfect, vague and difficult; hence, other scientists have a doubt about the validity of their chaos theory. However, they have no choice. Therefore, other scientists wait to appear as more advanced solutions than the old chaos theory.

Thus, this study presented a new solution herein; it seems an innovative scientific result. Surprisingly, in 2015, a non-physicist on the behalf of physicists found an analytical method, as shown in Table 1. Incomprehensibly, physicists do not welcome this new solution. Why do they unwelcome and displease it?

Because they already have the old solution such as chaos theory in statistical physics. If it is accepted, it is a disadvantageous solution to them like Copernican theory in Middle age. In other words, they assert that the new electric vehicle is not a formal car. Thus, this study will be described the reason in two parts, as below.

(Part 1: New Solution) The readers note Table 1, which was studied and achieved by non-physicists on the behalf of physicists through interdisciplinary research between physics and engineering. On the left side of Table 1, there is existing chaos theory in traditional physics, which is familiar to all scientists. However, the right side of the table describes a new solution for resolving nonlinear dynamics, as mentioned above. Amazingly, the above new solution was not resolved based on traditional physics but based on interdisciplinary research that it was first introduced the system analysis theory (see Figure 1(a)) [2] into nonlinear dynamics as follow; \{Control theory $\rightarrow$ Physical sciences\}. Unfortunately, it is outside of their academic framework of determinism; moreover, all scientists have not ever seen it before. Accordingly, most determinists unwelcomed it and they remained outsiders. However, there is no problem because the above mentioned new solution has nothing to do with determinists, so that physicists do not deny or criticize this study. On the other side, if the new solution in

Table 1. Comparison of the old logical solution and new systematical solution for closed loop systems in modern science (more detail of new solution will be described in Section 2).

\begin{tabular}{ccc}
\hline Division & Old Logical Solution & New Systematical Solution \\
\hline $\begin{array}{c}\text { Paradigm of } \\
\text { thinking }\end{array}$ & $\begin{array}{c}\text { Logical thinking } \\
\text { based on Determinism } \\
\text { How to solve } \\
\text { logical problem }\end{array}$ & $\begin{array}{c}\text { Systems thinking based on } \\
\text { Indeterminism + (Determinism) }\end{array}$ \\
$\begin{array}{c}\text { traditional physics } \\
\text { How to solve }\end{array}$ & $\begin{array}{c}\text { Solved by Chaos theory } \\
\text { based on logical thinking }\end{array}$ & The same as left solution \\
systematical problem & $\begin{array}{c}\text { Solved by systems } \\
\text { analysis theory }\end{array}$ \\
\end{tabular}




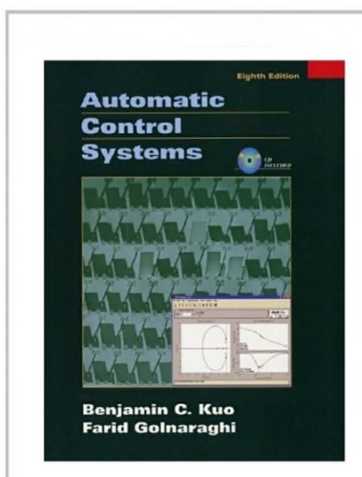

(a)

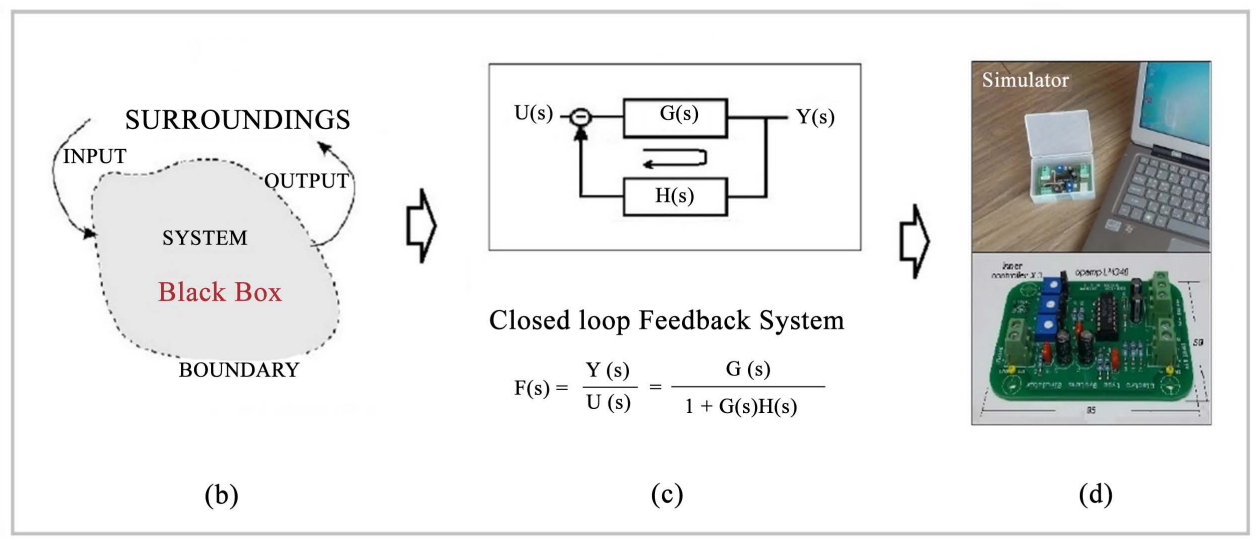

Figure 1. (a) Textbook on control theory; (b) basic system; (c) block diagram of internal mechanism of feedback system; (d) analog type simulator.

Table 1 is more perfect, precise, and easier than the previous chaos theory, non-physicists have no reason to follow their old solutions. The author encourages other scientists, including engineers, mathematicians, ecologists, and economists, to read the report [3] [4]. In addition to aid the readers' understanding, the main concept in Table 1 is following. First, the new solution was approached and achieved based on systems thinking [5]. Second, the above new solution was obtained via interdisciplinary research, which is described detail in Section 2.3.

(Part 2: To Non-physicists) Paradoxically, the new solution in Table 1 is similar to the heliocentric theory, thus, if it is true, it will be disadvantageous to them, hence, physicists are unwelcome it. In addition, there is the paradigms of scientific thinking, which is classified into \{logical thinking\} and ssystems thinking\}. However, classical and modern physicists have no idea about the systems thinking. For instance, in 2021, the author received an opinion from a physical journal S. Ironically, they misunderstood the above new solution as an epistemological problem; it does not make sense. It implies that no physicists have ability to review and evaluate this solution in Table 1, they are like outsiders because they firmly believe that there is no other solution than the chaos theory.

By contrast, other scientists have no reason to reject the new solution if the new solution is more perfect, precise, and easier than the old chaos theory; moreover, it will be applied to high-tech industries such as artificial intelligence (AI). However, the validity of above new solution can be confirmed by anyone with MATLAB program [6] or simulator as shown in Figure 1(d). Nevertheless, if physicists want to remain outsiders, regrettably, non-physicists have no reason to convince them. Then, they are involved in a truth game with non-physicists as the third party without their intention.

Meanwhile, if physicists avoided the new solution, the author has the following idea. First, the author attempts to separate it from physicists and move to other scientists in the third party, such as mathematicians, engineers. Next, they built a new academic curriculum as a second physics based on systems thinking, 
Finally, it will be launching into modern sciences. If they achieve it, they will cover the unsolved problems from nonlinear dynamics to metaphysical problems, which physicists have not resolved for a long time. It has not risk because other scientists have no obligation to follow determinists unless they clarify their problems.

(Summary) Finally, to prove the above description that the old chaos theory is imperfect and difficult, the author prepared four application examples in Section 2.4: 1) the famous Lorenz's butterfly effect, 2) random walk in the stock market, 3) logistic curve in ecosystems, and 4) Kuhn's innovation theory. Hence, nonlinear dynamics or complex systems must be resolved using the systematic solution based on systems thinking.

\section{Materials and Methods}

\subsection{Scientific Background}

In general, there are many unsolved problems in nonlinear dynamic systems, such as ecosystems, turbulence, climate change, and organisms. Most physicists misunderstood these problems as logical problems within the open-loop system. However, these problems can be classified into systematic problems within closed-loop systems with feedback, which can be resolved, based on systems thinking. This study aims to prove this. In addition, there are many metaphysical problems, e.g., "Why can we not predict daily stock prices?", "Why are ice ages and the food chain virtuous cycles?", and "Why do large-scale pandemics strike periodically?" Unfortunately, determinists cannot resolve the above questions using chaos theory. Why is it problem? Because they do not know how to analyze systematic problems within closed-loop systems, this study provides an example.

In 2008, the author have a question. Physicists who follow determinism treated \{logical problems\} and \{systematical problems\} as the same problems without distinction, further, they treated both problems as a black box as shown in Figure 1(b). Why do they so? Because determinists have no solutions for resolving nonlinear dynamics in that time. However, this is an inexact idea and a serious mistake in physics; moreover, this problem is shocking to the author. Unbelievably, no scientists knew to exist the analytic method of systems in other science as shown in Figure 1(a). Why didn't they know it? Unfortunately, the abovementioned analytic method was developed in the $20^{\text {th }}$ century more than about 300 years later.

If so, we need to review the systems analysis theory in Figure 1(a), which was developed for designing automatic systems during the Second World War. it is widely used as an engineering principle. Thus, the author on behalf of physicists will resolve the unsolved problems based on the paradigm of systems thinking here. Paradoxically, if classical physicists had known the systems analysis theory in Figure 1(a), they would have succeeded in resolving the nonlinear dynamic systematic problem mentioned above on behalf of the author. Regrettably, most 
physicists are intentionally avoiding this new solution in Table 1 . Since most determinists wish to stick with the old chaos theory.

\subsection{What Is Systems Thinking?}

In general, physical phenomena are classified into logical and systematic problems, and the paradigm of scientific thinking is divided into \{logical thinking\} and \{systems thinking\}. However, classical physicists in the $17^{\text {th }}$ century have no idea about the paradigm of systems thinking, because they have no choice. Unfortunately, there is no analytical method for resolving systematic problems such as the systems analysis theory (see Figure 1(a)) at that time. It is a very significant issue that it means that modern physics is not full-sized physics excluding the paradigm of systems thinking. Meanwhile, non-physicists, such as engineers and economists, are accustomed to the paradigm of systems thinking because they have to resolve various optimization problems based on systems thinking. If so, we need to know what is the system thinking and its analytic method as below.

(Mathematical Background) We need to note Figures 1(a)-(d). Classical physicists has treated all physical phenomena as the black box shown in Figure 1(b), and they are resolved it using logical solutions such as algebra and statistics based on logical thinking; in their academic framework. However, this is a serious misunderstanding. All physical phenomena in nature are treated as closed loop systems as shown in Figure 1(c) including open loop systems. If the feedback element $H(s)$ in Figure 1(c) is nothing (zero), it becomes open loop systems, which is logical problem such as classical mechanics. Hope the readers misunderstanding. Otherwise, if $H(s) \neq 0$, it is defined ad closed loop systems. [2].

For instance, by modeling the food chain as shown in Figure 2(a), it can be defined as a closed-loop system with feedback as shown in Figure 1(c), which contains two components such as predator's entropy $Q(s)$ and prey's entropy $H(s)$ [where $s$ is the Laplace operator], moreover, each components are related inversely as the time chart in Figure 2(b). Then, the numbers of predators and prey repeatedly increase and decrease endlessly according to time, moreover, converge to zero (equilibrium) and become steady. It is the internal mechanism

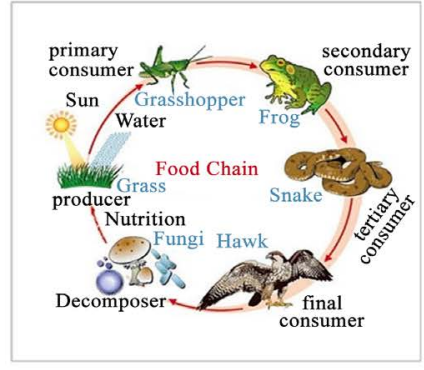

(a)

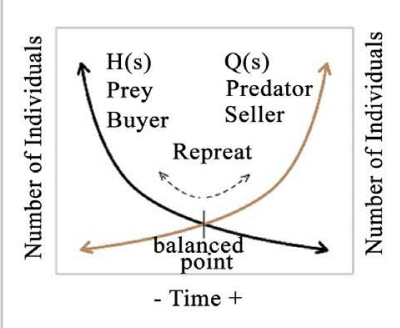

(b)

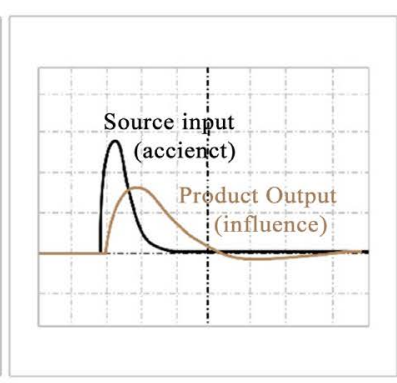

(c)

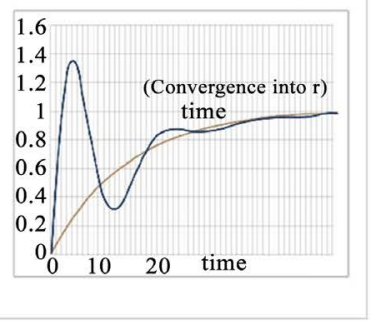

(d)

Figure 2. (a) food chain in ecosystem; (b) time chart of ecosystem - the relation between predator and prey; (c) the diagram of basic closed loop systems with feedback function is $F(s)=G(s) /(1+G(s) H(s))$; (d) initial phenomenon in complexity. 
of the food chain and its parameter $F(s)$ of food chain between the input and output is determined using Equation (1).

$$
F(s)=\frac{\text { output } Y(s)}{\text { input } U(s)}=\frac{G(s)}{1 \mp G(s) H(s)}
$$

Hence, we can analyze Equation (1) with system analysis theory in Figure 1 (a), thus, this was the mathematical background to systems thinking. In particular, the above example is the same as the scenarios of buyers and sellers in the stock market or laws of suppliers and demanders. In here, this study confirms the above reason why nonlinear dynamics cannot be resolved based on logical thinking using two books about complexity.

One is [Simply Complexity], written by a statistical physicist Neil Johnson [7], and the other is [Entropy], written by an economist Jeremy Rifkin [8]. They approached nonlinear dynamics involving complexity based on logical thinking with microscopic viewpoint. Nevertheless, they found only the external behavior of complexity such as irregularity, regularity, self-organization, and initial phenomenon, but could not find other characteristics such as no runaway, no overflow, and no explosive. Paradoxically, if they knew the new solution, they would not have written the books on complexity.

\subsection{Overview Mathematical Process: How to Resolve the Problems?}

This section describes the resolution of nonlinear dynamics using systems analysis theory, as mentioned above. If someone wants to resolve the problems, they can use an excellent computer program MATLAB [6], which does not require work by hand. Thus, the readers' attention is focused to the analog simulator in Figure 1 (d), which is able to reproduce the nonlinear dynamics in real time under virtual circumstances. Therefore, it can be resolved by anyone and anytime, moreover, it can be simulated any types of closed loop systems in nature. Hence, the research results may be confirmed by anyone using the above simulator at any time. Therefore, the readers need not to perform complex and detailed calculations.

However, here is an important reason as follows. Since all closed-loop systems cannot be calculated in reverse, hence, they must follow the following sequence: modeling-simulation-verification-return. Thus, if the readers cannot build the model system, as shown in Figure 1(c), they cannot go ahead. If they successfully work a modeling system, it can be resolved using MATLAB or simulation devices. For instance, if the closed loop systems in Figure 1(c) have been resolved manually, we obtain the output of Equation (2) as given below [2]. It is the same as the response of a unit function $u(t)$ in control engineering.

$$
y(t)=1-A \cdot \mathrm{e}^{-B \cdot t} \sin (W \cdot t+\varphi)
$$

where $t$ represents time, and $A, B, W$, and $\varphi$ are variable constants. The readers should note that Equation (2), which is a time series function in the system, is very important. Equation (2) explains the cause of creation of nonlinearity. To 
help the readers understand, assuming that the input source of the ecosystem is irregular, the output of the systems has nonlinearity, as shown in Figure 3(c), and butterfly effect. Notably, Equation (2) is very useful as it includes the damping factor $\beta$ (Remark; intrinsic variables that determine output characteristics). In addition, Equation (2) can easily be simulated by a simulation device that contains the same Equation (2). Therefore, anyone can reproduce the nonlinear dynamics using the simulation device or MATLAB.

\subsection{Application Example}

In the above Section, this study presented a new solution focused on the following idea; 1) it was achieved through interdisciplinary research; 2) it was resolved by a non-physicist on behalf of a physicist; 3 ) a new analytical method was revealed for them. To help the readers understanding, this study provides four application examples for readers, such as the butterfly effect, random walk, logistic curve, and innovation theory as below. Paradoxically, it is an evidence that proves the imperfect of the chaos theory.

\subsubsection{Redefined Lorenz's Butterfly-Effect}

In first case, the butterfly effect, it was first claimed in 1963 by Edward Norton Lorenz [9], who was not a physicist but a meteorologist. Observing that a small numerical variation $(0.506127>0.5016)$ during an iterative calculation process in a weather forecast led to a completely different result, he named it the butterfly effect, which appealed to the public and was welcomed by many determinists. Unfortunately, the author concluded that it was his misunderstanding as the initial phenomenon, as shown in Figure 2(d), resembled an impulse in an electrical system.

- (Modeling) However, he made a serious mistake in assuming that atmospheric entropy increases infinitely without saturation; hence, it is not explosive, no overflow and no runaway absolutely. The heat energy from the sun is moved to the polar region and exhausted to space based on the law of energy conservation. In this case, the mechanism can be converted into a basic model system based on systems thinking, as shown in Figure 1(c).

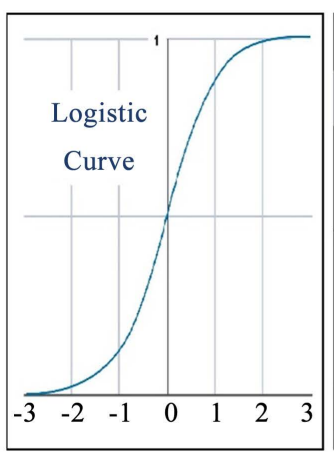

(a)

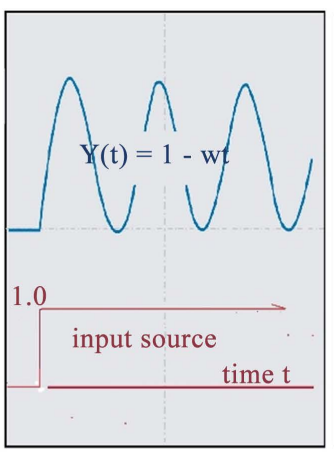

(b)

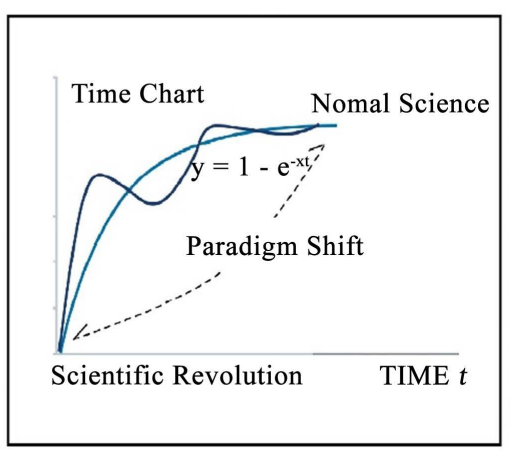

(c)

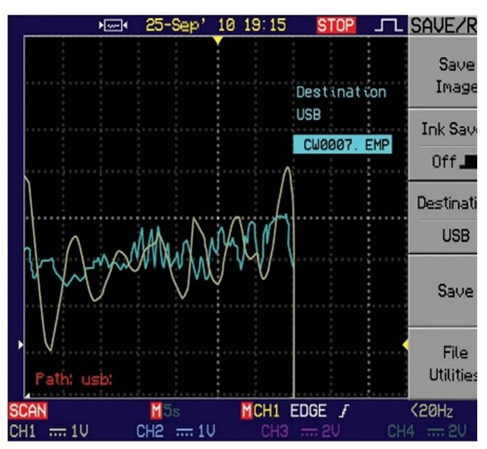

(d)

Figure 3. (a) Logistic curve; (b) simulation result: basic response of constant function (a periodicity as sine curve); (c) the scientific revolution time chart; (d) behavior of complexity from simulator such as daily stock price (Random walk). 
- (Simulation) Any scientist can then resolve it using MATLAB. The resulting behavior of the output $Y(s)$ is reproduced in Figure 2(d), which depicts a typical initial phenomenon with a closed-loop system that does not damp at all. It can be confirmed the references as Equation (1) refer to the textbook for details [3]; it is a decreasing periodic function. If the initial phenomenon is rapidly increasing (overshooting) in Figure 2(d), it easily disappears, similar to the butterfly effect. In similar cases, if the input source is an impulse, such as a lightning surge or accident, likewise, numerous disasters in history, such as the Great Depression, World War, and Financial Crisis, that have occurred but have been overcome by humans, the output is shown in Figure 2 (c), which is restored to the original state within a short period.

- (Verification) In conclusion, physicists should not exaggerate or magnify their assertions because no one trusts mysterious assertions. Paradoxically, had Lorenz known the above solution, he would not have made the assertion. Thus, this study classifies his assertion as fiction, which must be immediately eliminated from modern physics. Other scientists have to oust the mysterious physical laws.

\subsubsection{Redefined Random Walk in Stock Market}

- (Hypothesis) Physicists in Wall Street talked about the prediction of stock, which is a random walk [10]. The case of a stock market is like a thermodynamic system. The positive entropy of the seller desiring to sell at a high price is defined as $Q(s)$, while the negative entropy of the buyer desiring to buy at a low price is defined as $H(s)$, as shown in Figure $1(\mathrm{c})$. The two elements are inversely related to each other, and the out-put converges to zero state by time (remark; this is the same as heat in thermodynamics). Hence, we can define the correlation with the Laplace transform based on the systems analysis theory, as shown in Figure 1(c). It is the internal structure of the stock market.

- (Simulation) The above hypothesis can be easily resolved and proved using MATLAB or a specially designed analog simulator, as shown in Figure 1(d). In this case, the behavior of output is determined as Equation (2), but Equation (2) has a special variable such as the damping factor $\beta$, which is determined the external characteristics of stock market, as shown in Figure 3(d). Usually, the price fluctuates endlessly. Thus, physicists have named the characteristics of stock market as random walk. Absolutely, no one can predict the random walk such as stock price but it is not runaway, overflow and explosive. Paradoxically, if someone can find its parameter, he/she will succeed in the future.

- (Verification) Therefore, no one can predict the daily stock price. In conclusion, stock market systems are representative systematic problems. Therefore, the random-walk in stock market is a time-series function.

\subsubsection{Redefined Verhulst's Logistic Curve}

- (Hypothesis) It was asserted by the mathematician Verhulst [11], and it ex- 
plains why the increasing and decreasing population follows the sigmoid $\mathrm{S}$ curve, as shown in Figure 3(a). It can be proven through the food chain in an ecological system using the above solution. The number of predators and prey in the food chain are defined as $Q(s)$ and $H(s)$, respectively, in Figure 1(b). Each element was laid inversely, and convergence to equilibrium occurs over time. In this case, we can define the correlation, as shown in Figure $1(c)$. This can be solved using MATLAB.

- (Simulation) In the reference book, the output of all closed-loop systems is determined by Equation (2) above. For convenience, a detailed description is omitted. If the above model systems without any restriction, the output of the population is determined as $y(t)=1-\sin (\omega t)$. It is a periodic sine curve, as shown in Figure 3(b). It continues to increase and decrease repeatedly to follow the $S$ curve.

- (Verification) Thus, if one draws the equation without dimensions, we can obtain the sigmoid S, as shown in Figure 3(a). The sine curve can be easily reproduced using the simulator. Paradoxically, had Verhulst has known the above systematic solution, he would not have made the assertion. Hence, the logistic curve is a time series function.

\subsubsection{Tomas Kuhn's Scientific Revolution Structure}

Kuhn's innovation theory is a well-known philosophical principle that is well established in modern science. However, it is similar to the heliocentric theory developed in the Middle Ages. The scientific revolution encountered extreme opposition when it was first proposed, but it led to a paradigm shift, and the theory now widely accepted. Finally, it reached a saturated state as in the normal sciences. It has the following sequences: scientific revolution-paradigm shiftnormal science.

- (Modeling) all closed-loop systems, such as the food chain or stock market, have a time delay [d], because all dynamic physical phenomena slowly converge to saturation based on Equation (2). It can be summarized as is $y(t)=1-\mathrm{e}^{-x t}$ (where $x$ is constant). This equation describes a gradual increase and eventual saturation, as shown in Figure 3(c). This can be described by his assertion through the figure. It is realized in three steps: invention (innovation), paradigm shift, and saturation state (normal science).

- (Verification): As an example, mobile technology developed slowly for 150 years with the following sequence: invention-paradigm shift-normal science. The same is true for steam engine, gun powder, fertilizer, semiconductors, Internet, and mobile phone, whose utility slowly increased, converged into a saturated state, and then further into a normal state, as shown in Figure 3(c). Hence, Kuhn's theory is not a logical problem, but a systematic problem. If he had known the new solution, he would not have made the assertion.

\section{Result}

- There are many unsolved nonlinear dynamics problems, such as the food 
chain in ecosystems, within closed-loop systems and it is not yet clear.

- Physicists already have the old solution such as chaos theory in statistical physics, which is arranged by classical physicists based on logical thinking.

- However, it is a serious mistake because nonlinear dynamics is systematic problems that should be resolved it based on the paradigm of systems thinking.

- Hence, non-physicist on the behalf of physicist achieved a new systematic solution based on the systems thinking through an interdisciplinary research.

- Unfortunately, physicists unwelcome it because it is like Copernican theory and avoid cooperating with others. Hence, they themselves became outsiders.

- Therefore, non-physicists have no reason to follow their solution unless they clarify their problems in logical solution such as chaos theory.

\section{Discussions}

This thesis is a great achievement in modern science, it is resolved the unsolved problem based on systems thinking through interdisciplinary science, it is achieved by non-physicists on behalf of physicists as shown in Table 1. In addition, it has proved through experiments and application examples in Section 2.4. Unfortunately, the solution has nothing to do with physicists who stick to their chaos theory; they themselves became outsiders such as medieval astronomers.

Paradoxically, if other scientists would be mastered the new solution in the future, they would be covered many unsolved nonlinear dynamics problems and metaphysical problems in all sciences using the new solution in the second physics on behalf of physicists. If so, the above-mentioned truth game is over; the new electric vehicle is regular car. In addition, if other scientists understand what paradigm of systems thinking is, then, they can examine many mysterious principles. For instance, Lorenz's butterfly effect in statistical physics, they will reveal that Lorenz's assertion is proved a mysterious problem without an academic background but a time series function as shown in Figure 2(d).

In addition, the author, who wishes to establish the above-mentioned second physics as a formal modern science, has proposed this new solution to a well-known research organization, such as the Santa Fe Institute [12], in the USA. SFI's mission is to resolve the complexity in economics or ecology based on logical thinking by physicists on behalf of humanities scholars. Nevertheless, they cannot perform fulfill their mission because they approach systematic problems, such as macroeconomics and ecosystems, based on logical thinking; it is a mistake. Paradoxically, if they knew the new solution based on systems thinking mentioned above, they would have easily succeeded in resolving their work.

\section{Conclusions}

Most scientists have been known that physical phenomena were logical problems. However, there are many unsolved nonlinear dynamics involving complexity within closed-loop systems, such as the food chain, stock market, climate 
change, organisms, and turbulence. Classical physicists have been approached it based on logical thinking and re-solved using chaos theory in Table 1.

Meanwhile, this study is to demonstrate that nonlinear dynamics involving complexity are solvable problems based on system thinking, moreover, to present evidence in Section 2.4 that the old chaos theory based on logical thinking is imperfect, in addition, to prove that Lorenz's butterfly effect is invalidated as a mysterious problem. Nevertheless, determinists try to stick to their chaos theory; further, they do not want to be exposed their shortcomings to other scientists. If so, non-physicist cannot follow them unless physicists clarify the above problems.

In conclusion, the new solution will be established in modern science, and non-physicists can cover all nonlinear dynamics, including physical problems, using the second physics based on systems thinking. Therefore, the author encourages all scientists to introduce control theory into their disciplines. Hence, this study will contribute advance of science.

\section{Acknowledgements}

Eho technology co. research center supported research.

\section{Conflicts of Interest}

The authors declare no conflicts of interest regarding the publication of this paper.

\section{References}

[1] Casti, J.L. (1995) Complexification: Explaining a Paradoxical World through the Science of Surprise. Harper Perennial, New York.

[2] Golnaraghi, F. and Kuo, B.C. (1998) Automatic Control Systems. 10th Edition, McGraw-Hill Education, New York.

[3] Cha, D.S. (2015) Establishment of New Solution for Complex Systems in Multidiscip-Linary Science Based on Feedback System Analysis Method and Proven by Simulator. Journal of Modern Physics, 6, 1927-1934.

https://www.scirp.org/journal/paperinformation.aspx?paperid=60738 https://doi.org/10.4236/jmp.2015.613198

[4] Cha, D. and Jun, H. (2020) The Origin of Nonlinear Dynamics Involving Complexity in Modern Sciences. Open Journal of Applied Sciences, 10, 654-662. https://www.scirp.org/journal/paperinformation.aspx?paperid=103755 https://doi.org/10.4236/ojapps.2020.1010045

[5] Goodman, M. (2018) Systems Thinking: What, Why, When, Where, and How? https://thesystemsthinker.com/systems-thinking-what-why-when-where-and-how/

[6] MATLAB Simulink (2021) MathWorks Co. https://kr.mathworks.com/matlabcentral/?s_tid=gn_mlc

[7] Neil, J. (2020) Simply Complexity (2007 Korea Edition). Bada Publishing, Seoul.

[8] Jeremy, R. (1981) Entropy: A New World View. Bantam Books, London.

[9] Lorenz, E. (1963) Deterministic Non periodic Flow. Journal of the Atmospheric Sciences, 20, 130-141. 
https://journals.ametsoc.org/view/journals/atsc/20/2/1520-0469_1963_020_0130_dn f_2_0_co_2.xml https://doi.org/10.1175/1520-0469(1963)020<0130:DNF>2.0.CO;2

[10] Random walk (2020) Encyclopedia of Mathematics. https://encyclopediaofmath.org/wiki/Random_walk

[11] Linacre, L.J. (2009) Why Logistic (Sigmoid) Ogive and not Autocatalytic Curve? https://rasch.org/rmt/rmt64k.htm

[12] Santa Fe Institute (2021) https://santafe.edu/about 